\title{
Role of Advanced Endoscopic Imaging Techniques in the Management of Inflammatory Bowel Disease
}

\author{
Eun Soo Kim \\ Division of Gastroenterology and Hepatology, Department of Internal Medicine, Kyungpook National University School of Medicine, Daegu,
} Korea

Endoscopy plays a crucial role in the management of inflammatory bowel disease (IBD) in terms of diagnosis, monitoring of mucosal status, and surveillance of colitis-associated neoplasia. Mucosal healing evaluated by endoscopy has been recognized as the target of treatment in the era of powerful biologics therapy. The optimal modality for identifying dysplasia in IBD has yet to be well defined. Increasing progress has recently been made in endoscopic technologies to more accurately assess mucosal inflammation and more effectively detect dysplasia. Here we review the data of advanced endoscopic imaging techniques such as chromoendoscopy, virtual chromoendoscopy, endocytoscopy, and confocal laser endomicroscopy in the management of IBD. Clin Endosc 2017;50:424-428

Key Words: Chromoendoscopy; Endocytoscopy; Confocal laser endomicroscopy; Inflammatory bowel disease

\section{INTRODUCTION}

Inflammatory bowel disease (IBD), consisting of Crohn's disease (CD) and ulcerative colitis (UC), is characterized by chronic inflammation in the digestive tract and often requires lifelong management. The diagnosis of IBD is challenging, as there is no single gold standard diagnostic tool. It is usually established based on various evaluating modalities like clinical assessment, laboratory tests, endoscopy, radiologic evaluations, and histology. ${ }^{1-3}$ Among them, endoscopy is of paramount importance for making the correct diagnosis. In addition, endoscopy is crucial for the assessment of disease activity, which is required for proper therapeutic planning. Powerful biologic therapy has brought a paradigm shift in the therapeutic target from clinical symptoms to mucosal healing

Received: September 4, 2017 Revised: September 24, 2017

Accepted: September 24, 2017

Correspondence: Eun Soo Kim

Division of Gastroenterology and Hepatology, Department of Internal Medicine, Kyungpook National University School of Medicine, 130 Dongdeok-ro, Jung-gu, Daegu 41944, Korea

Tel: +82-53-200-5879, Fax: +82-53-200-5879, E-mail: dandy813@knu.ac.kr

(cc) This is an Open Access article distributed under the terms of the Creative Commons Attribution Non-Commercial License (http://creativecommons.org/ licenses/by-nc/3.0) which permits unrestricted non-commercial use, distribution, and reproduction in any medium, provided the original work is properly cited. that can be observed through endoscopy. Growing evidence shows that mucosal healing is associated with many favorable outcomes such as lower hospitalization rates, reduced disease relapse rates, and lower surgical rates. ${ }^{4,5}$ Therefore, the role of endoscopy in IBD is more essential now than before.

Patients with long-standing IBD are at an increased risk of colitis-associated colorectal cancer (CRC) ${ }^{6-8}$ Studies with longterm follow-up periods have shown that the 10-, 20-, and 30year incidences of CRC in patients with UC are $2 \%, 8 \%$, and $18 \%$, respectively, which are significantly higher than those in the general population. ${ }^{9}$ Thus, patients with IBD should undergo regular endoscopy to enable the early detection of CRC and precancerous lesion like dysplasia, leading to better prognosis. However, colitis-associated neoplastic lesions in patients with IBD are extremely difficult to find because they tend to be flat and indistinguishable against the background mucosa due to the surrounding chronic inflammation..$^{10,11}$

Recently, rapid advancements in endoscopic technologies including chromoendoscopy (CE), virtual CE, endocytoscopy, and confocal laser endomicroscopy (CLE) have enabled more accurate assessment of mucosal inflammation and more effective unveiling of dysplasia. In this review, we will discuss the role of these advanced endoscopic imaging techniques in the management in IBD and provide updated data in this field. 


\section{CHROMOENDOSCOPY}

Dye CE, which was introduced more than a decade ago, uses dye agents to enhance the detection of mucosal lesions. These agents include absorptive agents (methylene blue, toluidine blue, and cresyl violet), contrast agents (indigo carmine and acetic acid), and reactive staining agents (Congo red and phenol red). ${ }^{12}$ Among them, methylene blue and indigo carmine are the main agents for dye CE in IBD patients.

Dye CE shows a clear benefit for the detection of neoplasia in the context of IBD surveillance. Significantly more intraepithelial neoplastic lesions were found in the indigo carmineaided CE group than in the control group using magnification with conventional white-light endoscopy in 350 patients with long-standing UC (69 vs. $24, p<0.0001){ }^{13}$ Methylene blueassisted $\mathrm{CE}$ also reports a notable 3.2-fold increase in the dysplasia detection rate in UC compared with white-light endoscopy. ${ }^{14}$ In a prospective study, six endoscopists conducted white-light endoscopy followed by indigo carmine-based CE in 75 patients with UC. ${ }^{15}$ It showed a significantly better dysplasia detection rate $(21.3 \%$ vs. $9.3 \%, p=0.007)$ and similar high rate of interobserver agreement for polyp detection (kappa score 0.86 vs. 0.91 ) as compared with white-light endoscopy. Enhanced dysplasia detection of dye CE in UC was confirmed in a meta-analysis of six randomized controlled trials demonstrating a pooled sensitivity of $83 \%$, specificity of $91 \%$, and diagnostic odds ratio of $17.5 .^{16}$ According to these results, dye CE is currently recommended by most guidelines as an alternative to non-targeted random biopsies for dysplasia surveillance in long-standing UC. ${ }^{17,18}$ Dye CE also provides an accurate diagnosis of inflammation activity extent and severity in patients with UC. ${ }^{19,20}$

Despite the above advantages, dye CE is not accepted as an optimal technique in routine clinical practice due to the following limitations. ${ }^{21}$ First, it takes more time (dye CE increases procedure time by around $10 \mathrm{~min}$ ). Second, it requires operator training and the additional cost of the dye. Finally, there has yet to be a study demonstrating a significant advantage of using dye $\mathrm{CE}$ in terms of CRC-related morbidity and mortality.

Recent advances in endoscopic imaging technology allow the use of $\mathrm{CE}$ without spraying dye agents during colonoscopy. This dye-less $\mathrm{CE}$ or image enhanced endoscopy can be performed by just a click of a button with no need to apply specialized equipment in the middle of the procedure. Choices include narrow band imaging (NBI; Olympus, Tokyo, Japan), Fuji Intelligent Color Enhancement (FICE; Fujinon, Tokyo, Japan), and the i-scan (Pentax, Tokyo, Japan). NBI uses an optic filter that narrows down the spectrum of light emitted from the scope, resulting in better visualization of the muco- sal vascularity. ${ }^{22}$ FICE and the i-scan enhance images in different ways but use the same physical principle as NBI, which emphasizes the intensity of blue light. Instead of using optical filters inside of the endoscope, they digitally reconstruct virtual images in real time, using computed spectral estimation technology. ${ }^{23}$ Most studies were conducted using NBI.

Several studies have evaluated the role of NBI for predicting and detecting neoplasia in UC. A pilot study using magnifying endoscopy with NBI in 46 patients with UC showed the potential benefit of NBI to predict dysplasia. ${ }^{24}$ They analyzed the surface pattern and classified it into honeycomb $(n=161)$, villous $(n=85)$, and tortuous $(n=50)$. The positive rate of dysplasia was higher in the tortuous pattern than in other patterns ( $8 \%$ vs. $0.4 \%, p=0.003$ ) suggesting that the former recognized by NBI may predict the presence of dysplasia in UC. However, prospective studies failed to reveal a significant advantage of NBI in terms of surveillance of dysplasia in UC compared with white-light endoscopy. A randomized crossover study comparing white-light endoscopy with NBI in 48 patients with extensive UC for $>8$ years showed no significant difference in the detection of dysplasia between groups (NBI, 13 lesions vs. white-light endoscopy, 11 lesions, $p=0.727){ }^{25}$ In another randomized study of 112 patients with long-standing UC, there was no difference in dysplasia detection rates of 9\% in both arms. ${ }^{26} \mathrm{~A}$ recent randomized study including a large number of UC patients ( $n=159)$ also showed a comparable number of neoplasia with both techniques, indicating that NBI does not improve the detection of neoplasia in UC compared with white-light endoscopy. ${ }^{27}$ The only benefits of NBI over white-light endoscopy are fewer biopsy specimens and less withdrawal time. However, these prospective NBI studies did not use magnifying endoscopy. High magnification in combination with dye $\mathrm{CE}$ seemed to show a clear benefit over whitelight colonoscopy for detecting neoplastic lesions in UC. . $^{13,28}$ Therefore, a prospective study to evaluate the efficacy of magnifying NBI for colitis-associated dysplasia in UC is needed.

Several studies have determined the effect of dye-less CE compared with dye $\mathrm{CE}$ for the detection of neoplasia in IBD. ${ }^{29}$ -

${ }^{31}$ In a nutshell, NBI is not superior to dye CE for this purpose. A prospective crossover study comparing indigo carminebased CE with NBI for the detection of dysplasia in 60 IBD patients showed a higher neoplasia miss rate in the NBI group than in the dye CE group. ${ }^{31}$ Therefore, NBI cannot be recommended as the standard technique for surveillance in IBD. There is a lack of studies using FICE or the i-scan for detecting neoplasia in IBD patients.

Studies have shown a vital role of dye-less CE in the assessment of mucosal inflammation. In a study using magnifying NBI in 60 patients with IBD (17 CD and 43 UC) and 24 control participants, patients with CD and UC had branch- 
like structures more frequently and higher vascularity of the domes in Peyer's patches than control subjects. ${ }^{32}$ A randomized study comparing high-definition white-light endoscopy and the i-scan for detecting mucosal inflammation in $78 \mathrm{pa}-$ tients with IBD reported that the i-scan showed better agreement than white-light endoscopy with the histologic findings in extent $(92.3 \%$ vs. $48.7 \%)$ and degree $(89.7 \%$ vs. $53.9 \%)$ of inflammatory activity. ${ }^{33}$ Another study evaluated the value of NBI for assessing specific mucosal vascular patterns found in $67 \%$ of colorectal segments from 30 UC patients. ${ }^{34}$ Mucosal inflammation indicators such as acute inflammatory cell infiltrates, goblet cell depletion, and basal plasmacytosis were significantly more noted in patients with altered mucosal vascular pattern than in those with a normal pattern. Since mucosal healing is recognized as an important therapeutic endpoint in IBD, efforts have been made to evaluate mucosal healing status more accurately with enhanced endoscopic techniques. Very recently, a new endoscopic score for assessing the severity of mucosal inflammation in UC using the i-scan has been developed and validated, showing very good interobserver agreement. ${ }^{35}$ It defined and characterized the endoscopic mucosal and vascular healing, reflecting the full range of histologic changes. Taken together, these results indicate that dye-less $\mathrm{CE}$ can offer a precise assessment of mucosal inflammatory extent and severity of IBD patients.

\section{ENDOCYTOSCOPY}

Endocytoscopy is a novel technique enabling the real-time microscopic imaging of gastrointestinal mucosa with a magnification power up to 1400 -fold..$^{36}$ It is based on contact light microscopy along with preparation of the mucosal layer using absorptive contrast agents such as methylene blue or toluidine blue. In a pilot study of 40 IBD patients, endocytoscopy reliably differentiated single inflammatory cells with good sensitivity and specificity (neutrophils, $60 \%$ and $95 \%$; basophils, $74.4 \%$ and $94.4 \%$; eosinophilic granulocytes, $75 \%$ and $90.5 \%$; and lymphocytes, $88.9 \%$ and $93.3 \%$ ). ${ }^{37}$ Furthermore, the agreement rate between endocytoscopy and histology for grading intestinal inflammation was $100 \%$. However, the real clinical implication of this technique in IBD remains to be seen because data regarding this issue are still lacking. Further, there are no data on the role of endocytoscopy in the surveillance of colitis-associated neoplasia.

\section{CONFOCAL LASER ENDOMICROSCOPY}

CLE is also able to offer real-time in vivo histology of the intestinal mucosa with 1000 -fold magnification power during endoscopy. ${ }^{38}$ It requires a low-power blue laser that releases a 488-nm-wavelength light. The microscopic image is constructed based on this reflected light from the tissue. Before the examination, pretreatment consisting of the topical (cresyl violet or acriflavine) or systemic application (fluorescein) of fluorescence agents is required. ${ }^{39}$ One of the main differences between CLE and endocytoscopy is imaging plane depth. While endocytoscopy provides the very superficial mucosal layer with up to $50-\mu \mathrm{m}$ depth due to the contact light microscopy technique, CLE allows a deeper tissue analysis with up to $250-\mu \mathrm{m}$ depth. ${ }^{40}$

Many studies showed a promising role of CLE for the histologic assessment of mucosal inflammation in IBD. Colonic crypt tortuosity, an enlarged crypt lumen, microerosions, hypervascularization, and augmented mononuclear cell infiltrates were the main findings observed by CLE in active CD. ${ }^{41}$ CLE could detect mucosal pathologic abnormalities such as impaired and distorted crypt regeneration, persistent inflammation, and abnormal vascular patterns in UC patients with normal mucosa on white-light endoscopy. ${ }^{42}$ More importantly, clinical relapse in IBD could be predicted based on CLE analysis. A composite score using fluorescence leakage and crypt diameter detected by CLE predicted clinical relapse of UC in the following 1-year period. ${ }^{43}$ Another study revealed that ileal fluorescein leakage and microerosions on CLE were significant risk factors for flare-ups in $\mathrm{CD}^{44}$ Several studies determined the value of CLE for assessing intestinal barrier function in vivo. ${ }^{45-48}$ An increased gap density on CLE in the terminal ileum of patients with IBD was a significant predictor of poor clinical outcomes including flare-ups, hospitalization, or surgery in IBD. ${ }^{45,48}$

A recent study provided another encouraging role of CLE for molecular imaging in vivo. ${ }^{49}$ In 25 patients with $\mathrm{CD}$, the topical administration of a fluorescent anti-tumor necrosis factor (TNF) antibody was used to detect mucosal cells expressing membrane-bound TNF during CLE. Surprisingly, there was a significant difference in clinical response rates at week 12 after adalimumab therapy between patients with $\mathrm{CD}$ with many TNF-expressing cells and those with few or no TNF-expressing cells ( $92 \%$ vs. $15 \%, p=0.0002$ ). This result suggests a new potential of CLE in the field of molecular imaging for identifying therapeutic responses to biologics in IBD.

Regarding the detection of neoplasia in IBD, studies have failed to show a consistent benefit of CLE. ${ }^{50-52}$ Compared with NBI, CLE showed significantly low sensitivity for the diagnosis of neoplasia during surveillance colonoscopy in 22 patients with UC. ${ }^{53}$ A prospective study evaluating the diagnostic accuracy of CLE for detecting neoplastic lesions in 61 patients with $\mathrm{CD}$ reported similar accuracies of dye $\mathrm{CE}$ alone and dye 
Table 1. Evidence of the Potential Use of Advanced Endoscopic Imaging Techniques in IBD

\begin{tabular}{lcccc}
\hline & $\begin{array}{c}\text { Dye } \\
\text { chromoendoscopy }\end{array}$ & $\begin{array}{c}\text { Dye-less } \\
\text { chromoendoscopy }\end{array}$ & $\begin{array}{c}\text { Endocytoscopy } \\
\text { Confocal laser } \\
\text { endomicroscopy }\end{array}$ \\
\hline Dysplasia detection & +++ & - & - & + \\
Assessment of mucosal inflammation & ++ & +++ & ++ & - \\
$\begin{array}{l}\text { Prediction of clinical relapse } \\
\begin{array}{l}\text { Molecular imaging for therapeutic response } \\
\text { to biologics }\end{array}\end{array}$ & - & - & - & +++ \\
\hline
\end{tabular}

IBD, inflammatory bowel disease.

CE in combination with CLE ( $80.3 \%$ vs. $86.7 \%)$ but poor sensitivity in both groups ( $42.9 \%$ vs. $28.6 \%) .{ }^{52}$ It concluded that CLE had limited applicability, mainly due to frequent equipment failure. This failure of CLE as a surveillance strategy might be explained by several factors. First, the entire gastrointestinal tract cannot be covered by endomicroscopy because it only sees a limited field of view. Therefore, it is necessary to use other macroscopic techniques like CE for finding suspicious areas and targeted biopsy before using endomicroscopy. Second, it is not applicable for pedunculated or sessile lesions. And finally, high cost, long procedural time, and the need for additional equipment are obstacles to CLE use. More studies are needed to confirm the efficacy of CLE in daily practice.

\section{CONCLUSIONS}

The value of endoscopy in the management strategy of IBD is increasing. In the meantime, advanced endoscopic imaging technologies have almost revolutionized the role of endoscopy in IBD (Table 1). Dye CE is recognized as the gold standard tool for dysplasia surveillance in long-standing IBD. Dye-less CE approaches like NBI play a crucial role in the assessment of mucosal inflammation extent and severity in IBD. Endocytoscopy and CLE enable the real-time histologic evaluation of intestinal mucosa in vivo. CLE can reliably predict clinical relapse in patients with quiescent IBD. Further, CLE can be used for molecular imaging in the prediction of therapeutic response to biologics in IBD, indicating the potential of personalized medicine. The long-term efficacy, feasibility, and cost-effectiveness of these advanced techniques in daily routine practice remain to be discussed.

\section{Conflicts of Interest}

The author has no financial conflicts of interest.

\section{REFERENCES}

1. Mowat C, Cole A, Windsor A, et al. Guidelines for the management of inflammatory bowel disease in adults. Gut 2011;60:571-607.
2. Dignass A, Eliakim R, Magro F, et al. Second European evidence-based consensus on the diagnosis and management of ulcerative colitis part 1 : definitions and diagnosis. J Crohns Colitis 2012;6:965-990.

3. Lichtenstein GR, Hanauer SB, Sandborn WJ; Practice Parameters Committee of American College of Gastroenterology. Management of Crohn's disease in adults. Am J Gastroenterol 2009;104:465-483; quiz 464, 484.

4. Frøslie KF, Jahnsen J, Moum BA, Vatn MH; IBSEN Group. Mucosal healing in inflammatory bowel disease: results from a Norwegian population-based cohort. Gastroenterology 2007;133:412-422.

5. Colombel JF, Rutgeerts P, Reinisch W, et al. Early mucosal healing with infliximab is associated with improved long-term clinical outcomes in ulcerative colitis. Gastroenterology 2011;141:1194-1201.

6. Ullman TA, Itzkowitz SH. Intestinal inflammation and cancer. Gastroenterology 2011;140:1807-1816.

7. Rutter M, Saunders B, Wilkinson K, et al. Severity of inflammation is a risk factor for colorectal neoplasia in ulcerative colitis. Gastroenterology 2004;126:451-459.

8. Rubin DT, Huo D, Kinnucan JA, et al. Inflammation is an independent risk factor for colonic neoplasia in patients with ulcerative colitis: a case-control study. Clin Gastroenterol Hepatol 2013;11:1601-1608.e1-e4.

9. Eaden JA, Abrams KR, Mayberry JF. The risk of colorectal cancer in ulcerative colitis: a meta-analysis. Gut 2001;48:526-535.

10. Itzkowitz SH, Harpaz N. Diagnosis and management of dysplasia in patients with inflammatory bowel diseases. Gastroenterology 2004;126:1634-1648.

11. Tytgat GN, Dhir V, Gopinath N. Endoscopic appearance of dysplasia and cancer in inflammatory bowel disease. Eur J Cancer 1995;31A:11741177.

12. Neumann H, Mönkemüller K, Günther C, Atreya R, Vieth M, Neurath MF. Advanced endoscopic imaging for diagnosis of Crohn's disease. Gastroenterol Res Pract 2012;2012:301541.

13. Hurlstone DP, Sanders DS, Lobo AJ, McAlindon ME, Cross SS. Indigo carmine-assisted high-magnification chromoscopic colonoscopy for the detection and characterisation of intraepithelial neoplasia in ulcerative colitis: a prospective evaluation. Endoscopy 2005;37:1186-1192.

14. Kiesslich R, Fritsch J, Holtmann M, et al. Methylene blue-aided chromoendoscopy for the detection of intraepithelial neoplasia and colon cancer in ulcerative colitis. Gastroenterology 2003;124:880-888.

15. Picco MF, Pasha S, Leighton JA, et al. Procedure time and the determination of polypoid abnormalities with experience: implementation of a chromoendoscopy program for surveillance colonoscopy for ulcerative colitis. Inflamm Bowel Dis 2013;19:1913-1920.

16. Wu L, Li P, Wu J, Cao Y, Gao F. The diagnostic accuracy of chromoendoscopy for dysplasia in ulcerative colitis: meta-analysis of six randomized controlled trials. Colorectal Dis 2012;14:416-420.

17. Farraye FA, Odze RD, Eaden J, Itzkowitz SH. AGA technical review on the diagnosis and management of colorectal neoplasia in inflammatory bowel disease. Gastroenterology 2010;138:746-774, 774.e1-e4; quiz e12-e13.

18. Cairns SR, Scholefield JH, Steele RJ, et al. Guidelines for colorectal can- 
cer screening and surveillance in moderate and high risk groups (update from 2002). Gut 2010;59:666-689.

19. Ibarra-Palomino J, Barreto-Zúñiga R, Elizondo-Rivera J, Bobadilla-Díaz J, Villegas-Jiménez A. [Application of chromoendoscopy to evaluate the severity and interobserver variation in chronic non-specific ulcerative colitis]. Rev Gastroenterol Mex 2002;67:236-240.

20. Matsumoto T, Kuroki F, Mizuno M, Nakamura S, Iida M. Application of magnifying chromoscopy for the assessment of severity in patients with mild to moderate ulcerative colitis. Gastrointest Endosc 1997;46:400-405.

21. ASGE Technology Committee, Wong Kee Song LM, Adler DG, et al. Chromoendoscopy. Gastrointest Endosc 2007;66:639-649.

22. Kuznetsov K, Lambert R, Rey JF. Narrow-band imaging: potential and limitations. Endoscopy 2006;38:76-81.

23. Neumann H, Neurath MF, Mudter J. New endoscopic approaches in IBD. World J Gastroenterol 2011;17:63-68.

24. Matsumoto T, Kudo T, Jo Y, Esaki M, Yao T, lida M. Magnifying colonoscopy with narrow band imaging system for the diagnosis of dysplasia in ulcerative colitis: a pilot study. Gastrointest Endosc 2007;66:957-965.

25. van den Broek FJ, Fockens P, van Eeden S, et al. Narrow-band imaging versus high-definition endoscopy for the diagnosis of neoplasia in ulcerative colitis. Endoscopy 2011;43:108-115.

26. Ignjatovic A, East JE, Subramanian V, et al. Narrow band imaging for detection of dysplasia in colitis: a randomized controlled trial. Am J Gastroenterol 2012;107:885-890.

27. Leifeld L, Rogler G, Stallmach A, et al. White-light or narrow-band imaging colonoscopy in surveillance of ulcerative colitis: a prospective multicenter study. Clin Gastroenterol Hepatol 2015;13:1776-1781.e1.

28. Hurlstone DP, Sanders DS, McAlindon ME, Thomson M, Cross SS. High-magnification chromoscopic colonoscopy in ulcerative colitis: a valid tool for in vivo optical biopsy and assessment of disease extent. Endoscopy 2006;38:1213-1217.

29. Efthymiou M, Allen PB, Taylor AC, et al. Chromoendoscopy versus narrow band imaging for colonic surveillance in inflammatory bowel disease. Inflamm Bowel Dis 2013;19:2132-2138.

30. Sussman DA, Barkin JA, Martin AM, et al. Development of advanced imaging criteria for the endoscopic identification of inflammatory polyps. Clin Transl Gastroenterol 2015;6:e128.

31. Pellisé M, López-Cerón M, Rodríguez de Miguel C, et al. Narrow-band imaging as an alternative to chromoendoscopy for the detection of dysplasia in long-standing inflammatory bowel disease: a prospective, randomized, crossover study. Gastrointest Endosc 2011;74:840-848.

32. Hiyama S, Iijima H, Shinzaki S, et al. Narrow band imaging with magnifying endoscopy for Peyer's patches in patients with inflammatory bowel disease. Digestion 2013;87:269-280.

33. Neumann H, Vieth M, Günther C, et al. Virtual chromoendoscopy for prediction of severity and disease extent in patients with inflammatory bowel disease: a randomized controlled study. Inflamm Bowel Dis 2013;19:1935-1942.

34. Kudo T, Matsumoto T, Esaki M, Yao T, Iida M. Mucosal vascular pattern in ulcerative colitis: observations using narrow band imaging colonoscopy with special reference to histologic inflammation. Int J Colorectal Dis 2009;24:495-501.

35. Iacucci M, Daperno M, Lazarev M, et al. Development and reliability of the new endoscopic virtual chromoendoscopy score: the PICaSSO (Paddington international virtual chromoendoscopy score) in ulcerative colitis. Gastrointest Endosc 2017 Mar 18 [Epub]. https://doi.org/10.1016/ j.gie.2017.03.012.
36. Neumann H, Fuchs FS, Vieth M, et al. Review article: in vivo imaging by endocytoscopy. Aliment Pharmacol Ther 2011;33:1183-1193.

37. Neumann H, Vieth M, Neurath MF, Atreya R. Endocytoscopy allows accurate in vivo differentiation of mucosal inflammatory cells in IBD: a pilot study. Inflamm Bowel Dis 2013;19:356-362.

38. Kiesslich R, Burg J, Vieth M, et al. Confocal laser endoscopy for diagnosing intraepithelial neoplasias and colorectal cancer in vivo. Gastroenterology 2004;127:706-713.

39. Neumann H, Kiesslich R, Wallace MB, Neurath MF. Confocal laser endomicroscopy: technical advances and clinical applications. Gastroenterology 2010;139:388-392, 392.e1-e2.

40. Iacucci M, Panaccione R, Ghosh S. Advances in novel diagnostic endoscopic imaging techniques in inflammatory bowel disease. Inflamm Bowel Dis 2013;19:873-880.

41. Neumann H, Vieth M, Atreya R, et al. Assessment of Crohn's disease activity by confocal laser endomicroscopy. Inflamm Bowel Dis 2012;18:2261-2269.

42. Macé V, Ahluwalia A, Coron E, et al. Confocal laser endomicroscopy: a new gold standard for the assessment of mucosal healing in ulcerative colitis. J Gastroenterol Hepatol 2015;30 Suppl 1:85-92.

43. Buda A, Hatem G, Neumann H, et al. Confocal laser endomicroscopy for prediction of disease relapse in ulcerative colitis: a pilot study. J Crohns Colitis 2014;8:304-311.

44. Karstensen JG, Săftoiu A, Brynskov J, et al. Confocal laser endomicroscopy: a novel method for prediction of relapse in Crohn's disease. Endoscopy 2016;48:364-372.

45. Kiesslich R, Duckworth CA, Moussata D, et al. Local barrier dysfunction identified by confocal laser endomicroscopy predicts relapse in inflammatory bowel disease. Gut 2012;61:1146-1153.

46. Liu JJ, Wong K, Thiesen AL, et al. Increased epithelial gaps in the small intestines of patients with inflammatory bowel disease: density matters. Gastrointest Endosc 2011;73:1174-1180.

47. Turcotte JF, Wong K, Mah SJ, et al. Increased epithelial gaps in the small intestine are predictive of hospitalization and surgery in patients with inflammatory bowel disease. Clin Transl Gastroenterol 2012;3:e19.

48. Shavrov A, Kharitonova AY, Davis EM, et al. A pilot study of confocal laser endomicroscopy to predict barrier dysfunction and relapse in pediatric inflammatory bowel disease. J Pediatr Gastroenterol Nutr 2016;62:873-878.

49. Atreya R, Neumann $\mathrm{H}$, Neufert $\mathrm{C}$, et al. In vivo imaging using fluorescent antibodies to tumor necrosis factor predicts therapeutic response in Crohn's disease. Nat Med 2014;20:313-318.

50. Günther U, Kusch D, Heller F, et al. Surveillance colonoscopy in patients with inflammatory bowel disease: comparison of random biopsy vs. targeted biopsy protocols. Int J Colorectal Dis 2011;26:667-672.

51. Freire P, Figueiredo P, Cardoso R, et al. Surveillance in ulcerative colitis: is chromoendoscopy-guided endomicroscopy always better than conventional colonoscopy? A randomized trial. Inflamm Bowel Dis 2014;20:2038-2045.

52. Wanders LK, Kuiper T, Kiesslich R, et al. Limited applicability of chromoendoscopy-guided confocal laser endomicroscopy as daily-practice surveillance strategy in Crohn's disease. Gastrointest Endosc 2016;83:966-971.

53. van den Broek FJ, van Es JA, van Eeden S, et al. Pilot study of probebased confocal laser endomicroscopy during colonoscopic surveillance of patients with longstanding ulcerative colitis. Endoscopy 2011;43:116122. 